\title{
A contribuição da estimulação psicomotora para o processo de independência do deficiente visual
}

\author{
The contribution of a psychomotor stimulation to the process of \\ independence for a visually impaired
}

\author{
F.A. Soares, T.R. Silva, D.P. Gomes, E.T. Pereira
}

ARTIGO ORIGINAL | ORIGINAL ARTICLE

\begin{abstract}
O presente estudo objetivou elaborar e aplicar um programa de estimulação psicomotora baseado no processo de orientação e mobilidade do deficiente visual. Este tipo de comprometimento influencia diretamente o desenvolvimento psicomotor do indivíduo e condiciona a autonomia e independência do mesmo. O programa foi aplicado no Laboratório de Estimulação Psicomotora da Universidade Federal de Viçosa, com o embasamento teórico da Psicomotricidade. O indivíduo estudado possuia um diagnóstico médico de grave problema visual desde a infância e optou-se pelo estudo de caso intrínseco, para observar e avaliar melhor dificuldades encontradas, mediante a técnica da observação sistematizada e entrevista informal com os pais. Pôde-se perceber evoluções no deficiente visual estudado, no que tange ao equilíbrio, noção espacial e esquema corporal e também na disposição em realizar atividades da vida diária, o que interferiu diretamente na sua locomoção. Concluiu-se que o processo de orientação e mobilidade é primordial no trabalho de independência do deficiente visual e que este necessita de um estímulo ao desenvolvimento psicomotor desde o início da sua vida, visto os atrasos que poderão surgir durante o seu crescimento e maturação, devido a possíveis dificuldades com o meio interno e externo gerados pelo comprometimento citado. Torna-se importante a aplicação de novos estudos com amostras maiores que se utilizem também abordagens qualitativas.
\end{abstract}

Palavras-chave: deficiência visual, estimulação psicomotora, orientação, mobilidade

ABSTRACT

This study aimed at developing and implementing a program of psychomotor stimulation process-based orientation and mobility of the visually impaired, since this type of commitment has a direct influence on psychomotor development of the individual, affecting their autonomy and independence. The program was implemented in the Laboratory of Psychomotor Stimulation of the Federal University of Viçosa, with the theoretical basis of Psychomotricity. We chose the intrinsic case study, to observe and evaluate better the difficulties encountered by the technique of systematic observation and informal interviews with parents. One can perceive evolutions in visually handicapped studied in terms equilibrium, concept space and body schema, and also willing to perform activities of daily living, which interfere in their locomotion. We concluded that the process of orientation and mobility is paramount in the work of independence of the visually impaired, and that it is needed a psychomotor development stimulus since the beginning of his life, because the delays that may arise during their growth and maturation. It becomes important to apply qualitative approaches for further studies but with larger samples. Keywords: visual impairment, psychomotor stimulation, orientation, mobility

Submetido: 08.09.2011 | Aceite: 02.12.2012

Felipe Alves Soares, Thaynara Rodrigues da Silva, Dênia Paula Gomes e Eveline Torres Pereira. Universidade Federal de Viçosa, Minas Gerais, MG, Brasil.

Endereço para correspondência: Felipe Alves Soares, Departamento de Educação Física/UFV, 36570-000 Viçosa, MG, Brasil.

E-mail: felipeefi_ufv@hotmail.com 
O último censo demográfico do Instituto Brasileiro de Geografia e Estatística indicou, dentre as várias pesquisas realizadas, a existência de mais de 25 milhões de brasileiros que possuem algum tipo de deficiência. Traçando um panorama mundial, o Brasil ocupa o sétimo lugar em um ranking que estabelece proporções de pessoas com deficiência (a cada 100 brasileiros, cerca de 15 compõem esse grupo) na população. No quadro dos diversos tipos de deficiência física, sensorial ou cognitiva, aparece em destaque a deficiência visual (DV) e, no Brasil, existem hoje mais de 15 milhões de deficientes visuais. A DV compreende desde pequenas alterações na acuidade visual até a ausência de percepção de luz, mas as alterações que têm implicações mais sérias para a vida das pessoas com necessidades especiais e para as suas famílias são a baixa visão e a cegueira (Laplane \& Batista, 2008).

A cegueira é definida como a perda total da visão até a ausência de projeção de luz. Já a baixa visão é definida como uma perda severa, sendo a acuidade visual inferior a 20/60 ou campo visual inferior a 10 graus do seu ponto de fixação, de forma que não há como realizar correção por meio do uso de óculos convencionais, por tratamento clínico nem mesmo por intervenção cirúrgica (Santos, Passos, \& Rezende, 2007).

Existe uma interação muito próxima entre visão e desenvolvimento, de forma que a diminuição das capacidades visuais implica uma série de comprometimentos em outras áreas do comportamento, dentre elas a área motora. A fim de se evitar o surgimento de comprometimentos nessa área, percebeu-se a necessidade de estimular o deficiente visual (DV) e permitir que o mesmo tenha um desenvolvimento adequado. Esse panorama propicia a aplicação de trabalhos de estimulação, como os que se baseiam na metodologia denominada Psicomotricidade, que será o alicerce teórico para aplicação deste trabalho. Meur e Staes (1984) a definem como uma corrente que permite rela- cionar três vertentes: a motricidade, a mente e a afetividade, que posteriormente facilitará a abordagem global do indivíduo por meio da técnica da observação sistematizada.

\section{Estimulação psicomotora e DV}

O processo de crescimento e desenvolvimento do indivíduo é marcado por comportamentos específicos para cada fase de maturação. Estas fases, determinadas pelas diferentes escalas, como a de Gallahue (2005), traduzem ações, que refletem as formas com que cada um dos indivíduos vê o mundo e o seu corpo e progride em relação à idade. Padronizou-se aquilo que certa idade faz ou não e a partir daí se vêem quais são os estímulos necessários para se chegar a este padrão e quais as interferências que condicionam o não fazer.

Vários fatores podem ser relacionados como influenciadores deste sistema de desenvolvimento humano. Maturação neural, problemas na transmissão de estímulo neural, deficiências ou poucos estímulos e ainda os elementos culturais podem ser citados como exemplos desses fatores que influenciam o desenvolvimento, tanto para além quanto para aquém do considerado "normal".

A articulação entre motricidade, cognição e afetividade centra-se na idéia de que os conceitos básicos de aprendizagem (dentro/ fora, grande/pequeno, etc.) são experimentados primeiramente no corpo do sujeito para que depois passem a ser representados. Sendo assim, quando o indivíduo se depara com um conflito, ele usará os esquemas que já dispõe para resolvê-los, e estes esquemas são, em sua maioria, esquemas psicomotores, esquemas corporais.

Dessa maneira, são definidos alguns fundamentos básicos para o trabalho de estimulação psicomotora: esquema corporal (essencial na formação da identidade); orientação espaço-temporal (possibilidade de exercer as capacidades corporais); equilíbrio (manter-se sobre uma base reduzida de sustentação do corpo); 
coordenação (tanto de habilidades motoras grossas quanto finas); lateralidade (a dominância lateral diz respeito a dados neurológicos, mas pode ser influenciada por hábitos sociais).

A visão possui um papel chave no desenvolvimento motor, pois coloca o indivíduo em contato com a realidade externa e fornece estímulos que auxiliam na orientação e no controle da ação corporal. Grande parte das experiências na exploração do meio, na descoberta do próprio corpo e na relação com os outros tem uma participação direta da visão, que se torna um elemento chave na organização da ação motora voluntária, consciente e segura. A diminuição severa da visão, portanto, implica uma situação oposta, que coloca o indivíduo em uma séria situação de risco: a falta de estímulos pode acarretar déficits em funções nas quais o DV, a priori, não possui nenhum tipo de comprometimento, destacando a importância do acompanhamento precoce nessa condição (Santos et al., 2007).

Os comprometimentos acarretados por essa deficiência podem ser de cunho primário (a nível orgânico) ou cunho secundário (a nível psicossocial), sendo este último o foco deste trabalho, que se refere a dificuldades de significação de sensações corporais, construção de pistas ambientais, autoconhecimento do corpo e reconhecimento do ambiente externo. Cabe destacar, a partir daí, atrasos na construção do esquema corporal (Meur e Staes (1984) definem esquema corporal como um elemento básico indispensável para a formação da personalidade da criança, uma representação relativamente global, científica e diferenciada que a criança tem de seu próprio corpo), devido à perda de elementos da comunicação não-verbal (posturas, gestos e expressões faciais) e da noção espacial, visto que os cegos normalmente têm capacidade limitada em relação às habilidades de imagens espaciais (Postma, Zuidhoek, Noordzij, \& Kappers, 2008).

Normalmente, os comprometimentos secundários surgem com maior intensidade, devido às barreiras físicas, educacionais e atitudinais impostas pela sociedade para a participação social e cultural da pessoa com DV. Esses comprometimentos a nível psicossocial podem acontecer de forma mais intensa, porque são mediados socialmente e estão relacionados ao fato do universo cultural estar construído em função de um padrão de normalidade (Nuernberg, 2008). Os comprometimentos secundários, consequentemente, geram atrasos e déficits no desenvolvimento do DV, que podem interferir nos ganhos de autonomia e independência, dadas as dificuldades encontradas no desenvolvimento do esquema corporal e da noção espacial. Paralelamente a esse aspecto, surge a necessidade de trabalhos específicos sobre orientação e mobilidade do indivíduo, pois, de acordo com Melo (1991), esses itens são os principais elementos na construção da independência do DV.

\section{Orientação e mobilidade}

A orientação é definida por Melo (1991) como um processo em que o DV usa de outros sentidos para o estabelecimento de suas posições em relação com todos os objetos significativos do seu meio circundante. A mobilidade, por sua vez, é a capacidade de deslocamento do ponto em que se encontra o indivíduo para alcançar outra zona desse meio.

Santin e Simmons (1977) relatam que, para o DV, a mobilidade é necessária para se estabelecer um elo primário com o mundo exterior, um elo sem o qual ficaria prejudicado o seu desenvolvimento auditivo e tátil. O DV tem pouca evidência da estrutura do espaço que o rodeia até poder movimentar-se no sentido de descobrir essa evidência. Santos (2005) mostra que os sentimentos de autoestima e interação estão relacionados com a independência física e a capacidade de movimentar-se livremente leva a uma melhor participação e a um maior reconhecimento dos demais cidadãos.

Lamônica et al. (2008) descrevem como as barreiras arquitetônicas interferem na vida 
do DV, podendo deixá-lo segregado da convivência e vida social e que este é um fator que fere o direito de acessibilidade dos mesmos. Com este panorama, existe uma crescente demanda por profissionais com capacidade de desenvolver trabalhos especializados e com competências para atuar junto a esse público da sociedade. A partir disso, percebe-se que o professor de Educação Física poderia ser um agente importante na aplicação de um trabalho em prol da orientação e mobilidade do DV. O foco se dá no resgate da funcionalidade e independência na vida do mesmo, através de exercícios para marcha, coordenação motora, lateralidade, orientação espaço-temporal, equilíbrio, esquema corporal, itens que caracterizam os fundamentos da Psicomotricidade.

Nesta perspectiva, o objetivo deste estudo foi elaborar um programa de estimulação psicomotora para um DV, visando a locomoção e mobilidade do mesmo. A partir da sua execução, o DV terá maiores oportunidades de desenvolver-se adequadamente e alcançar sua independência e autonomia.

\section{Caracterização do indivíduo}

O participante desta pesquisa, aqui referido como L, do sexo masculino, 23 anos de idade, $1.62 \mathrm{~m}$ e $64 \mathrm{~kg}$, vivia na cidade de Viçosa/MG. O diagnóstico médico do oftalmologista dizia que L tinha um grave problema visual desde a infância. Seus resíduos visuais o permitem enxergar apenas vultos e feixes luminosos de cores em ambos os olhos $(<20 / 1600$ na Snellen em AO).

De acordo com Dantona e Dantona (citado por Salomão, Mitsuhiro, e Belfort, 2009), níveis de acuidade visual $<20 / 400$ ou $<20 / 200$, no melhor olho, são a referência para se definir a cegueira, sendo que a DV é determinada pela acuidade visual de $20 / 60$ ou menos, referente ao melhor olho. Nas perspectivas desses autores, L poderia ser caracterizado como cego, tendo em consideração o seu nível de acuidade visual. O diagnóstico médico apontava ainda para a presença de ceratocone (doença não-inflamatória degenerativa do olho, que pode causar distorção substancial da visão, múltiplas imagens, raios e sensibilidades à luz, que são freqüentemente relatados pelos pacientes) em ambos os olhos, tendo havido transplante de córnea do olho esquerdo, devido à degeneração ter sido mais grave. Esse processo cirúrgico teve consequências, desencadeando uma crise de epilepsia meses após. A crise resultou num traumatismo e o transplante teve que ser refeito em caráter emergencial.

O quadro atual de L mostra que ele é afácico (falta do cristalino), tem corectopia OE (situação anormal da pupila no olho esquerdo, não se encontrando no centro da íris) e nistagmo (movimentos oculares oscilatórios, rítmicos e repetitivos dos olhos). Com os aspectos citados, o diagnóstico médico concluiu não haver, no momento, um caminho que permita a recuperação da visão.

Associado à DV, L apresenta um quadro de esquizofrenia e hipertensão arterial, fazendo atualmente uso dos seguintes medicamentos: Carbamazepina $200 \mathrm{mg}$ (Anticrise convulsiva, remédio utilizado para o tratamento da epilepsia), Haloperidol $5 \mathrm{mg}$ (controle da agressividade), Risperidona $2 \mathrm{mg}$ e Clorpomazina $100 \mathrm{mg}$ (ambos para tratamento da esquizofrenia), Biperideno $50 \mathrm{mg}$ (utilizado para evitar reações adversas extrapiramidais induzidas pelos neuroléticos), Melleril $50 \mathrm{mg}$ (aplicado na terapêutica nervosa) e Captopril $25 \mathrm{mg}$ (tratamento da hipertensão arterial).

Com relação às características motoras, $\mathrm{L}$ possui passadas curtas, tanto pela insegurança que a deficiência pode trazer, bem como pela dificuldade na coordenação motora, e, além disso, anda com rotação externa do quadril em ambos os lados. L consegue realizar apenas algumas atividades básicas e intermediárias da vida diária, AVD's (Cotton, 1998), como, por exemplo, vestir-se e pentear-se, sendo que não consegue arrumar sua própria cama e preparar suas refeições. Entretanto, esses aspectos que 
dizem respeito à sua capacidade funcional estão ligados não só à cegueira, mas também ao seu cotidiano excessivamente protegido pela família, quadro que o tornou uma pessoa dependente, afirmação feita a partir de diálogos com os pais, que relatam a dependência de $\mathrm{L}$ em arrumar a própria cama, preparar suas refeições, organizar seus utensílios pessoais, etc.. Esse comportamento perdura pelo papel de mãe superprotetora que interfere negativamente no processo de independência do filho, sendo que as entrevistas e conversas informais constantes com a mãe reafirmaram esse panorama. No que diz respeito aos comprometimentos sociais, $\mathrm{L}$ não possui dificuldades de interação e consegue se comunicar muito bem.

Pode-se inferir, através das observações, atrasos significativos no que tange ao seu desenvolvimento cognitivo. Mesmo aos 23 anos de idade, $L$ não consegue realizar somas simples de Matemática ou mesmo reconhecer letras do alfabeto. Possivelmente, isso poderia ter acontecido devido à falta de acessibilidade, dos quatro aos 11 anos de idade, a uma escola adaptada e adequada às suas deficiências e pela ausência de estimulação, conforme relatado pela mãe.

\section{MÉTODO}

\section{Amostra}

A aplicação desta pesquisa deu-se no Laboratório de Estimulação Psicomotora (LEP) situado no Departamento de Educação Física da Universidade Federal de Viçosa (UFV). Todo o procedimento foi aprovado pelo Comitê de Ética em Pesquisas com Seres Humanos da Universidade Federal de Viçosa, atendendo às orientações da Resolução 196/96 do CNS de 10/10/96 sobre experimentos com seres humanos.

No primeiro encontro preencheu-se uma ficha cadastral com a mãe de L, contendo informações como dados gerais (nome, endereço, etc.), tipo de comprometimento (através de exames e diagnósticos trazidos pelos pais), histórico médico e familiar, se possui alguma doença (se sim, qual), medicamentos tomados, como é a alimentação diária, desempenho nas AVD`s (tomar banho sozinho, se alimentar, vestir-se e pentear-se, etc.). Esse primeiro contato serviu para se estabelecer um diálogo entre professor e aluno e para que o primeiro pudesse compreender os comprometimentos e necessidades do indivíduo a ser analisado. Após isso, deu-se o primeiro atendimento, onde atividades de cada tema da Psicomotricidade foram aplicadas. A partir da observação desse primeiro momento da estimulação, traçou-se a periodização específica do aluno.

Para o desenvolvimento deste estudo, optou-se pelo uso do método denominado como estudo de caso intrínseco definido por Stake (citado por Mazzoti, 2006) como:

"Aqui, o estudo não é empreendido primariamente porque o caso representa outros casos ou porque ilustra um traço ou problema particular, mas porque, em todas as suas particularidades e no que tem de comum, este caso é de interesse em si. O pesquisador, pelo menos temporariamente, subordina outras curiosidades para que as histórias dos que "vivem o caso' emerjam."

Gil (1987) caracteriza o estudo de caso como um estudo profundo, exaustivo, de um ou de poucos objetos, de maneira a permitir conhecimento amplo e detalhado do mesmo. Essas características explicam o porquê da escolha do estudo de caso intrínseco, visto ainda que ele é recomendável na investigação de temas polêmicos. Como mostra May (2004), o trabalho se dá com necessidades que são "típicas" em algum sentido ou são tomadas como "críticas" naquilo em que são diferentes.

O planejamento da intervenção foi realizado a partir da estimulação psicomotora e do registro de observação sistematizada, bem como a análise do comportamento motor do DV em questão, englobando os seguintes aspectos: relações do DV com o seu próprio corpo, com o espaço, o tempo, o objeto e o outro. Para tanto, 
o olhar focou-se sobre a expressividade motora do indivíduo, visando identificar dificuldades e atrasos no seu desenvolvimento psicomotor, para a posterior elaboração de um programa de estimulação psicomotora.

Os itens a serem observados durante as sessões de estimulação foram subdivididos de acordo com o fundamento psicomotor: lateralidade (homogênea, ambidestra ou cruzada), noção espaço-temporal (sucessão de acontecimento, noção de tempo e noção de ritmo), esquema corporal (sentido cinestésico, auto-imagem, imitação de gestos e reconhecimento de direita/esquerda e frente/atrás) coordenação motora (grossa e fina) e equilíbrio (estático e dinâmico).

A técnica da observação citada acima permite flexibilidade na pesquisa e, como explicado por May (2004), ajuda a transpor a lacuna entre o entendimento dos estilos de vida alternativos das pessoas e os preconceitos com que a diferença e a diversidade defrontam-se com tanta frequência.

Ao fim de cada sessão de estimulação, o professor elaborou relatórios que serviram como instrumentos de registro dessa observação e avaliação do aluno, focando nos itens psicomotores a serem observados e pautando-se em quatro tópicos: tipo de comprometimento, consequências observáveis desse comprometimento no desenvolvimento motor, cognitivo e social, respostas às atividades desenvolvidas e comentários pessoais sobre a dinâmica da aula e comportamento do aluno.

Foi recolhido também, semanalmente, um relato escrito pelos pais do DV, onde todas as melhorias conseguidas com o Projeto e verificadas no lar foram explicitadas, o que caracterizou uma entrevista informal com o pesquisador. Optou-se pelo uso desse tipo de entrevista pela possibilidade de oferecer uma visão mais próxima do problema pesquisado.

Os métodos e técnicas dessa pesquisa foram utilizados visando respeitar a individualidade do participante, pois no LEP a avaliação não se dá através de comparações a padrões, mas sim na evolução em relação a si próprio. Caracterizou-se, assim, a avaliação referenciada a si próprio, que requer a interpretação do resultado do indivíduo comparando com o resultado anterior do mesmo.

Essa proposição tende a superar qualquer noção da pessoa com deficiência em referência ao pressuposto da normalidade, bem como valorizar a melhora qualitativa dos gestos motores do individuo, que não são assimilados por técnicas de coletas quantitativas (Nuernberg, 2008).

Com os aspectos metodológicos citados, os atendimentos com o DV em questão deram-se durante três meses, com duração de 50 minutos cada sessão e frequência de uma vez por semana, perfazendo um total de 11 encontros.

\section{Procedimentos}

Para aplicar o trabalho de estimulação psicomotora com L, realizaram-se 11 encontros, sendo o primeiro utilizado para analisar os principais déficits e, dessa forma, estabelecer os temas das 10 aulas posteriores, a fim de se permitir uma evolução gradativa na sua independência. A periodização deu-se da seguinte forma:

- Semana 1: Primeiros contatos (através de diálogos e preenchimento da ficha cadastral com a responsável, no caso a mãe) e trabalhos com atividades para cada fator da Psicomotricidade (observação dos possíveis atrasos do aluno);

- Semana 2: Equilíbrio (banco sueco, trave de equilíbrio, plataforma de equilíbrio e circuito com obstáculos - primeiramente o DV realizava o primeiro contato com o aparelho - intervenções cinestésicas a todo o momento por parte do professor);

- Semana 3: Esquema corporal e lateralidade (quebra-cabeça corporal e "jogos com bola de guizo" - uso deste material como adaptação, trazendo um estímulo sonoro ao aluno); 
- Semana 4: Marcha (pés de EVA - intervenções cinestésicas a todo o momento) e circuito com obstáculos - nessa atividade o professor modificava o posicionamento dos objetos a cada repetição executada por L. Além disso, teve início nessa atividade a implementação do uso de uma bengala adaptada: um cabo de vassoura. Esse objeto passou a ser utilizado para que $\mathrm{L}$ rastreasse o ambiente, e assim, pudesse evoluir no que diz respeito à sua independência. A técnica de Hoover foi utilizada como propõe Melo (1991), em que a bengala deve ser tomada de maneira firme: nem frouxa, de tal maneira que fique solta dentro da mão, nem apertada demais, a ponto de 'rebater', golpeando o solo com dureza e chamando a atenção dos circunstantes, pelo ruído produzido no solo;

- Semanas 5 e 6: Orientação e mobilidade (Parte principal do trabalho - caminhadas no Departamento de Educação Física da UFV, com orientações sobre o uso da bengala);

- Semanas 7 e 8: Orientação e mobilidade e orientação espacial (parte principal do trabalho - caminhadas no Departamento de Educação Física da UFV, com orientações sobre o uso da bengala - no fim da aula, o professor montou um circuito e constantemente mudava a ordem dos objetos, sendo que $L$ tinha que perceber essas mudanças e estabelecer a rota mais adequada);

- Semana 9: Orientação e mobilidade (parte principal do trabalho - caminhadas no Departamento de Educação Física da UFV, com orientações sobre o uso da bengala - estabelecimento de percursos diferentes e mais complexos, com a inclusão de escadas, áreas não conhecidas anteriormente e caminhadas em volta de uma piscina) e cognição (boliche adaptado com bola de guizo - o objetivo principal era trabalhar de forma concreta as somas matemáticas com o aluno, sendo que cada pino tinha o valor de um ponto);

- Semana 10: Cognição (boliche adaptado e tapete abecedário de EVA - reconhecimento das letras através da percepção tátil);
- Semana 11: Equilíbrio (mesmas atividades da semana 2, a fim de verificar alguma possível evolução no comportamento de L).

As atividades de percepção tátil e esquema corporal foram realizadas para que L aprendesse a relacionar os seus outros sistemas-guia, como o tato, a audição e o olfato, com os objetos significativos presentes no ambiente.

Castro, Paula, Tavares, e Moraes (2004) descrevem sobre a necessidade de um trabalho cognitivo apurado nesse processo, pois além da informação sensorial abrangente na orientação espacial, o uso da cognição enquanto uma função para a construção de mapas espaciais internos acaba sendo uma espécie de interface entre os fatores intrínsecos e extrínsecos nas tarefas de orientação.

\section{RESULTADOS E DISCUSSÕES}

$\mathrm{Na}$ primeira semana de trabalho com $\mathrm{L}$, observou-se, durante a sua caminhada até o laboratório, um comportamento dependente em relação à sua mãe. Esse foi mais um fator relevante no estabelecimento da orientação e mobilidade como foco deste trabalho. Silva e Dessen (2001) mostraram que o ambiente possui uma riqueza de elementos para o desenvolvimento do indivíduo, principalmente a sua característica mutável, dinâmica e cheia de confrontos. Se este é privado na exploração do ambiente, terá prejuízos durante o seu desenvolvimento e é justamente esse o panorama do caso L, onde em muitas situações L foi poupado e protegido de situações desafiadoras e estressantes. Bastos e Deslandes (2008) revelaram a falta de conhecimento dos pais em relação à deficiência dos filhos, sendo talvez essa uma importante explicação para o excesso de zelo dos cuidadores.

Com relação à intervenção psicomotora aplicada, as observações permitiram perceber alguns resultados positivos nesses três meses. No que diz respeito ao desenvolvimento motor, L apresentou evoluçõe no equilíbrio, mostrando maior independência na execução das ativi- 
dades na semana 11 em relação à semana dois (os desequilíbrios foram reduzidos e tornaram-se menos acentuados, ao passo ainda que no início do trabalho L só realizava a atividade com o auxílio do professor), na noção espacial e esquema corporal, aliado à compreensão de informações como "de costas" e "lateralmente", através da vivência e experiência cinestésica de como o corpo posiciona-se nessas situações, sendo que $\mathrm{L}$ teve mais dificuldades no que diz respeito à coordenação motora, talvez pelo curto período de intervenção. Os relatos transmitidos pelos pais demonstraram que essa intervenção psicomotora trouxe, numa análise em curto prazo, melhoras também no que diz respeito ao convívio social e essencialmente na disposição de realizar atividades da vida diária (AVD's).

Powers (2002) descreve em seu trabalho que o maior desafio é o de promover uma visão ampla de inclusão como um sistema compartilhado de valores e, em seguida, para trabalhar com os demais recursos de reavaliação e melhorar as práticas na educação de alunos especiais. Já Zaborowski (citado por Hayeems, Geller, Finkelstein, \& Faden, 2005) aprovou uma metodologia qualitativa baseada na auto-estima e na forma de enxergar a própria identidade, visto que a cegueira requer mudanças cognitivas. Eles concluíram demonstrando a necessidade de integração e que os ajustes necessários implicam mudanças no comportamento a partir da reconstrução da identidade pessoal.

Castro et al. (2004) relataram resultados positivos no seu estudo sobre aspectos da orientação espacial de participantes com DV, que podem evoluir após um treinamento com a navegação independente, através de atividades físicas, jogos esportivos, recreação, dança e atividades aquáticas. Os resultados encontrados nesses trabalhos corroboram as melhorias obtidas com $\mathrm{L}$, onde a estratégia foi estabelecer uma interrelação entre a estimulação psicomotora em prol da orientação e mobili- dade do participante e a mudança atitudinal do mesmo, principalmente no que diz respeito à busca de autonomia e independência.

Kallie, Scharater, e Legge (2007) analisaram as variantes e mudanças de direção na marcha de indivíduos cegos, que apresentam instabilidade, por exemplo, no andar em linha reta e em manter uma estabilidade na marcha. Eles relataram ainda a possibilidade de assimetrias físicas e biomecânicas e a presença de comportamentos em espiral durante a caminhada.

Com relação à biomecânica da marcha de L, a mesma foi caracterizada anteriormente como uma deambulação de amplitude curta e com rotação externa de quadril. Durante as atividades de orientação e mobilidade constantemente foram passados comandos verbais e cinestésicos para o aumento da amplitude da passada, a fim de se retirar a insegurança constante durante a caminhada e evitar possíveis assimetrias físicas e biomecânicas.

\section{CONCLUSÕES}

O uso do método de estudo de caso e da técnica da observação, como proposto por May (2004) e Gil (1987), permitiu uma flexibilidade na pesquisa realizada e proporcionou uma maior compreensão do indivíduo estudado. Com a intervenção psicomotora realizada, L pôde receber auxílios para a sua orientação, que permitiram desenvolver, organizar e melhorar o entendimento das relações espaciais básicas, principalmente (Lamônica et al., 2008). Todos esses mecanismos são essenciais para a locomoção em ambientes não-familiares. Percebeu-se que o processo de orientação e mobilidade é primordial no trabalho de independência do DV e que este necessita de um estímulo ao desenvolvimento psicomotor como base, visto os atrasos que podem surgir durante o seu crescimento e maturação. Algumas limitações foram enfrentadas na pesquisa, como no uso da bengala adequada. Adaptou-se a bengala, com a utilização de um cabo de vassoura e diante da técnica de Hoover, como proposta por Melo 
(1991). Não é o ideal, mas tornou-se uma importante adaptação para o desenvolvimento do seu processo de independência. Optou-se em qualificar os ganhos (pela observação) e torná-las funcionais ao seu cotidiano, ao invés de quantificar as evoluções apresentadas. Este estudo permitiu verificar também a necessidade de serem realizadas pesquisas com amostras maiores, sempre com o respeito das individualidades e que não ocorram comparações a padrões, dando-se maior relevância ao uso do método avaliativo referenciado a si próprio. A presente pesquisa, de caráter exploratório, possibilitou ainda a elaboração de uma perspectiva interna do fenômeno estudado e, futuramente, as análises poderão ser extrapoladas para casos similares.

\section{Agradecimentos:}

Nada declarado.

\section{Conflito de Interesses:}

Nada declarado.

\section{Financiamento:}

Este estudo é oriundo da pesquisa: "Análise da estimulação psicomotora voltada ao deficiente através de um instrumento de observação", financiada pela FUNARBE - Fundação Arthur Bernardes.

\section{REFERÊNCIAS}

Bastos, O. M., \& Deslandes, S. F. (2008). A experiência de ter um filho com deficiência mental: Narrativas de mães. Caderno de Saúde Pública, 24(9), 2141-2150.

Castro, E. M., Paula, A. I., Tavares, C. P., \& Moraes, R. (2004). Orientação espacial em adultos com deficiência visual: Efeitos de um treinamento de navegação. Psicologia: Reflexão e Crítica, 17(2), 199-210.

Cotton, E. (1998). Exercise for older adults: ACE's guide for fitness profissionals. Champaign: Human Kinetics.

Gallahue, O. (2005). Compreendendo o desenvolvimento motor de bebês, crianças, adolescentes e adultos. São
Paulo: Phorte.

Gil, A. C. (1987). Métodos e Técnicas de Pesquisa Social. São Paulo: Atlas.

Hayeems, R. Z., Geller, G., Finkelstein, D., \& Faden, R. R. (2005). How patients experience progressive loss of visual function: A model of adjustment using qualitative methods. British Journal of Ophthalmology, 89 (5), 615-620.

Kallie, C., Scharater, P. R., \& Legge, G.E. (2007). Variability in stepping direction explains the veering behavior of blind walkers. Journal of Experimental Psychology: Human Perception and Performance, 33(1), 183-200.

Lamônica, D. A. C., Araújo Filho, P., Simonelli, S. B. J., Caetano, V. L. S., Regina, M. R. R., \& Regiani, D. M. (2008). Acessibilidade em ambiente universitário: identificação de barreiras arquitetônicas no campus da USP de Bauru. Revista Brasileira de Educação Especial, 14(2), 177-188.

Laplane, A. L. F., \& Batista, C. G. (2008). Ver, não ver e aprender: A participação de crianças com baixa visão e cegueira na escola. Caderno CEDES, 28(75), 209-227.

May, T. (2004). Pesquisa social: Questões, métodos e processos (C. A. S. N. Soares, Tradução). Porto Alegre: Artmed.

Mazzotti, A. J. A. (2006). Usos e abusos dos estudos de caso. Caderno de Pesquisas, 36 (129), 637-651.

Melo, H. F. R. (1991). Deficiência visual: lições práticas de orientação e mobilidade. Campinas: Editora da Unicamp.

Meur, A., \& Staes, L. (1984). Psychomotricité et Reéducation (A. M. I. Galuban \& S. ONO, Tradução). São Paulo: Manole.

Nuernberg, A, H. (2008). Contribuições de Vigotski para a educação de pessoas com deficiência visual. Psicologia em Estudo, 13(2), 307-316.

Postma, A., Zuidhoek, S., Noordzij, M. L., \& Kappers, A. M. L. (2008). Keep an eye on your hands: on the role of visual mechanisms in processing of haptic space. Cognitive Processing, 9(1), 63-68.

Powers, S. (2002). From concepts to practice in deaf education: A United Kingdom Perspective on Inclusion. Journal of Deaf Studies and Deaf Education, 7(3), 230-243. 
Salomao, S. R., Mitsuhiro, M. R. K. H., \& Belfort Junior, R. (2009).Visual impairment and blindness: an overview of prevalence and causes in Brazil. Anais da Academia Brasileira de Ciências. 81(3), 539-549.

Santin, S., \& Simmons, J. N. (1977). Problemas das crianças portadoras de deficiência visual congênita na construção da realidade. Recuperado de http:// www.ibc.gov.br/?itemid=95\#more

Santos, A. (2005). O cego, o espaço, o corpo e o movimento: uma questão de orientação e mobilidade. Recuperado de http://www.ibc.gov.br/?itemid=106\#more
Santos, L. C., Passos, J. E. O. S., \& Rezende, A. L. G. (2007). Os efeitos da aprendizagem psicomotora no controle das atividades de locomoção sobre obstáculos em crianças com deficiência da visão. Revista Brasileira de Educação Especial, $13(3), 365-380$.

Silva, N. L. P., \& Dessen, M. A. (2001). Deficiência Mental e Família: Implicações para o Desenvolvimento da Criança. Psicologia: Teoria e Pesquisa, $17(2), 133-141$.

(c) EY-NC Todo o conteúdo da revista Motricidade está licenciado sob a Creative Commons, exceto quando especificado em contrário e nos conteúdos retirados de outras fontes bibliográficas. 